\title{
mRNA deadenylation and telomere disease
}

\author{
Philip J. Mason and Monica Bessler
}

Division of Hematology, Department of Pediatrics, The Children's Hospital of Philadelphia, Philadelphia, Pennsylvania, USA.

\begin{abstract}
Dyskeratosis congenita (DC) is an inherited BM failure disorder that is associated with mutations in genes involved with telomere function and maintenance; however, the genetic cause of many instances of DC remains uncharacterized. In this issue of the $J C I$, Tummala and colleagues identify mutations in the gene encoding the poly(A)-specific ribonuclease (PARN) in individuals with a severe form of DC in three different families. PARN deficiency resulted in decreased expression of genes required for telomere maintenance and an aberrant DNA damage response, including increased levels of $p 53$. Together, the results of this study support PARN as a DC-associated gene and suggest a potential link between $\mathrm{p} 53$ and telomere shortening.
\end{abstract}

\section{Poly(A)-specific ribonuclease $(P A R N)$ is a new DC gene}

In this issue, Tummala et al. identify a DC-associated gene that has no known connection with telomere metabolism (14). Specifically, Tummala and colleagues performed whole exome sequencing on a cohort of patients with severe DC, which is classified as Hoyeraal Hreidarsson syndrome (HHS) and manifests at an early age with immunodeficiency, intrauterine growth deficiency, and cerebellar hypoplasia. Their analysis revealed the presence of mutations in PARN, which encodes a poly(A)-specific ribonuclease (15). In 22 unrelated individuals, biallelic mutations in PARN that were likely to impair function were identified in three families. In these families, the affected children all presented with the HHS phenotype, and statistical analysis suggested that this was extremely unlikely to have occurred by chance, indicating that mutations in PARN underlie these cases of HHS. In one family, a brother and a sister were both homozygous for a missense mutation that results in an $\mathrm{A}$ to $\mathrm{V}$ substitution at amino acid 393 (PARN ${ }^{\text {A393V }}$ ), which is in a conserved $\mathrm{N}$-terminal domain of the protein that is essential for nuclease activity. In a second family, the individual with HHS was homozygous for a point mutation that abolishes a donor splicing site in the PARN transcript, and analysis of mRNA in blood from this patient revealed an absence of properly spliced PARN RNA and the presence of two different PARN transcripts, one of which was missing one exon and the other two exons. In a third family, the affected child was a compound heterozygote at the PARN locus and harbored one allele with a single base insertion that resulted in a frameshift and one allele with an insertion in a donor splice site that was predicted to abolish splicing. Moreover, compared to healthy, agematched controls, affected individuals in two of the families had substantially shorter telomeres. Telomere length was not measured in the affected individual from the third family. Together, the identification of PARN mutations in multiple fami- 

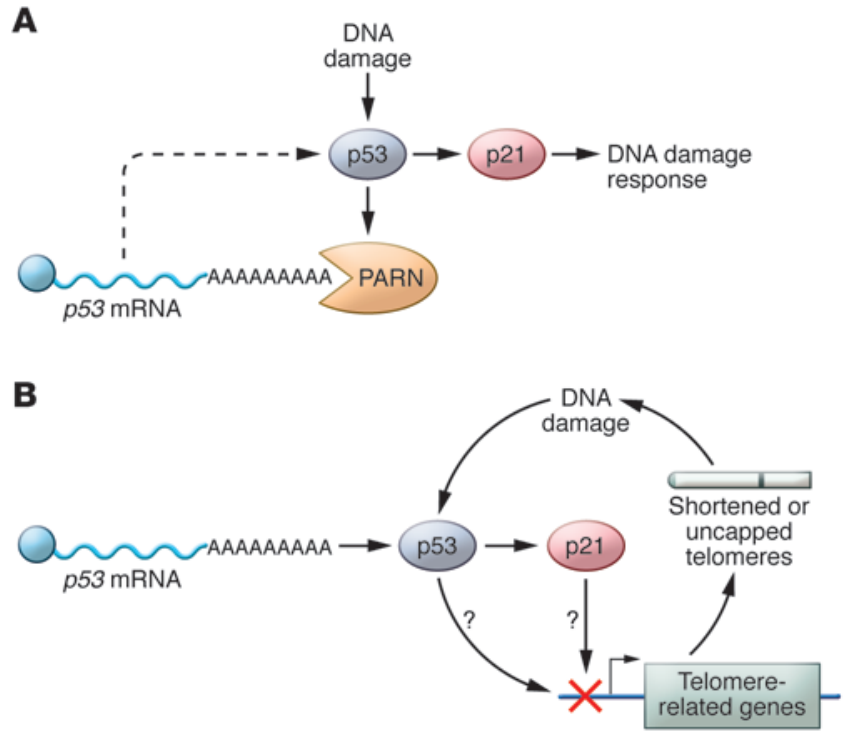

lies with cases of HHS and the observed decrease in telomere length in affected individuals strongly support $P A R N$ as a DC-associated gene.

It is not clear how PARN would affect telomere maintenance. PARN is a poly(A)specific deadenylase that regulates gene expression by shortening poly(A) tails on transcripts, thereby decreasing their stability. Recent studies indicate that PARN activity is differentially regulated under various cellular conditions $(16,17)$. Tummala and colleagues demonstrated that cells from individuals with $P A R N$ mutations had reduced deadenylation activity, which should affect transcript stability. While PARN has been extensively studied at the biochemical level, the range of mRNA substrates and the physiological relevance of PARN-targeted transcripts has not been fully characterized. Tummala and colleagues compared expression of genes involved in telomere maintenance in the blood of healthy controls and individuals with PARN mutations. They found decreased expression of several key regulators of telomere metabolism, including DKC1, TERF1, RTEL, and TERC. The same transcripts were also decreased in HEK293T cells treated with PARN siRNA (14).

\section{p53: guilty by association?}

Tummala et al. also observed that PARNdeficient patient cells exhibit an abnormal DNA damage response, including increased cell death and an increase in p53 following UV exposure (14). PARN has recently been shown to participate in positive and negative feedback loops that mediate p53 and mRNA processing (18). In nonstressed conditions, PARN deadenylates and destabilizes p53 mRNA through recognition of a sequence element in the 3'UTR. Under DNA damaging conditions, such as UV exposure, p53 accumulates, associates with, and activates PARN by forming a complex that includes cleavage stimulation factor 1 (CSTF1). Based on this model, a DNA damage-associated increase in p53 would subsequently be kept in check by PARN-dependent destabilization of $p 53$ mRNA; therefore, loss of PARN activity would lead to an increase in $p 53$ mRNA levels. Activation of p53 has been observed in a variety of BM failure syndromes, including Fanconi anemia (19, 20), Diamond Blackfan anemia (21), and even some forms of DC $(22,23)$. Recently, Simeonova et al. (24) described a strain of mice that is homozygous for a Trp53 allele that results in production of a truncated p53, which lacks $31 \mathrm{C}$-terminal amino acid residues. In these animals, both the truncated p53 and transcripts of p53 targets Cdkn1a, which encodes $\mathrm{p} 21$, and oncogene $M d m 2$ were increased. Most of these mice died 14-43 days after birth and exhibited DC-associated features, including BM failure, dark skin, cerebellar hypoplasia, and pulmonary fibrosis. Transcript analysis in embryonic fibroblasts from these animals also revealed a decrease in transcripts associated with telomere maintenance, including Dkc1, Rtel1, Tinf2, and Terf1,
Figure 1. Possible effects of PARN depletion on telomeres. (A) DNA damage in replicating WT cells causes increased levels of the tumor suppressor p53, which in turn induces p21 and the DNA damage response. p53 also activates the PARN deadenylase, which destabilizes p53 mRNA, thereby reducing p53 levels (17). (B) In the absence of PARN, p53 levels can increase unchecked and lead to inhibition of telomere-related genes and short telomeres. Shortened telomeres exacerbate the DNA damage response, further increasing $p 53$ levels, and thereby activating a cycle that leads to short telomeres and cell cycle arrest in rapidly replicating cells - a hallmark of DC pathobiology.

which overlap with some of the telomereassociated genes Tummala et al. (14) determined to be downregulated in PARNdeficient cells.

Together, these studies suggest a potential mechanism for the development of DClike phenotypes in the chronic absence of PARN (Figure 1). In rapidly dividing PARNdeficient cells, p53 levels would rise in response to an accumulation of DNA damage. The lack of PARN deadenylase activity would allow accumulation of p53 mRNA and further increase protein levels. Elevated p53 would, in turn, decrease transcription of genes required for telomere maintenance, with a subsequent shortening of telomeres. A novel feature in this model is the regulation of p53 at the mRNA level, whereas p53 is typically regulated at the level of protein stability. Such a mechanism would result in a cycle that enhances telomere shortening and increases p53, thereby inducing failure of tissues that are renewed via stem cell expansion. More work will need to be done to validate the involvement of the p53 pathway in aberrant telomere maintenance in PARN-associated DC; however, if correct, this model would provide an alternative pathway by which aberrant p53 activation contributes to the development of disease manifestations such as BM failure. Further investigation of the relationship between telomere metabolism, p53, and PARN may provide crucial information about BM failure and the prevalence of leukemic transformation and other forms of cancer in these disorders (13). 


\section{Acknowledgments}

P.J. Mason's work is supported in part by NIH/NCI grant R01 CA106995, and M. Bessler's work is supported in part by NIH/ NCI grant R01 CA105312 and by the Buck Family Chair in Hematology.

Address correspondence to: Philip J. Mason, Leonard and Madlyn Abramson, Room 302E, Pediatric Research Center, The Children's Hospital of Philadelphia, 3615 Civic Center Boulevard, Philadelphia, Pennsylvania 19104-4318, USA. Phone: 267.426.9327; E-mail:masonp@email.chop.edu.

1. Zinsser F. Atrophia Cutis Reticularis cum Pigmentations, Dystrophia Unguium et Leukoplakis oris (Poikioodermia atrophicans vascularis Jacobi.) Ikonographia Dermatologica. 1910;5:219-223.

2. Connor JM, Gatherer D, Gray FC, Pirrit LA Affara NA. Assignment of the gene for dyskeratosis congenita to Xq28. Hum Genet. 1986;72(4):348-351.

3. Knight SW, et al. 1.4 Mb candidate gene region for $\mathrm{X}$ linked dyskeratosis congenita defined by combined haplotype and $\mathrm{X}$ chromosome inactivation analysis. JMed Genet. 1998;35(12):993-996.

4. Heiss NS, et al. X-linked dyskeratosis congenita is caused by mutations in a highly conserved gene with putative nucleolar functions. Nat Genet. 1998;19(1):32-38.

5. Lafontaine DL, Bousquet-Antonelli C, Henry Y, Caizergues-Ferrer M, Tollervey D. The box $\mathrm{H}+\mathrm{ACA}$ snoRNAs carry Cbf5p, the putative rRNA pseudouridine synthase. Genes Dev. 1998;12(4):527-537.

6. Watkins NJ, et al. Cbf5p, a potential pseudouridine synthase, and Nhp2p, a putative RNA-binding protein, are present together with Garlp in all $\mathrm{H} \mathrm{BOX}$ / ACA-motif snoRNPs and constitute a common bipartite structure. RNA.1998;4(12):1549-1568.

7. Mitchell JR, Wood E, Collins K. A telomerase component is defective in the human disease dyskeratosis congenita. Nature. 1999;402(6761):551-555.

8. Vulliamy T, et al. The RNA component of telomerase is mutated in autosomal dominant dyskeratosis congenita. Nature. 2001;413(6854):432-435.

9. Armanios M, et al. Haploinsufficiency of telomerase reverse transcriptase leads to anticipation in autosomal dominant dyskeratosis congenita. Proc Natl Acad Sci U S A. 2005;102(44):15960-15964.

10. Vulliamy TJ, Walne A, Baskaradas A, Mason PJ, Marrone A, Dokal I. Mutations in the reverse transcriptase component of telomerase (TERT) in patients with bone marrow failure. Blood Cells Mol Dis. 2005;34(3):257-263.

11. Gramatges MM, Bertuch AA. Short telomeres: from dyskeratosis congenita to sporadic aplastic anemia and malignancy. Transl Res. 2013;162(6):353-363.

12. Guo Y, et al. Inherited bone marrow failure associated with germline mutation of ACD, the gene encoding telomere protein TPP1. Blood. 2014;124(18):2767-2774.

13. Alter BP, et al. Malignancies and survival patterns in the National Cancer Institute inherited bone marrow failure syndromes cohort study. $\mathrm{Br}$ J Haematol. 2010;150(2):179-188.

14. Tummala H, et al. Poly(A)-specific ribonuclease deficiency impacts telomere biology and causes dyskeratosis congenita. J Clin Invest. 2015;125(5):2151-2160.

15. Buiting K, Korner C, Ulrich B, Wahle E, Horsthemke B. The human gene for the poly(A)-specific ribonuclease (PARN) maps to $16 \mathrm{p} 13$ and has a truncated copy in the Prader-Willi/Angelman syndrome region on $15 \mathrm{q} 11 \rightarrow \mathrm{q} 13$. Cytogenet Cell Genet. 1999;87(1-2):125-131.

16. Cevher MA, et al. Nuclear deadenylation/ polyadenylation factors regulate 3 ' processing in response to DNA damage. $E M B O J$. 2010;29(10):1674-1687.

17. Lee JE, Lee JY, Trembly J, Wilusz J, Tian B, Wilusz CJ. The PARN deadenylase targets a discrete set of $\mathrm{mRNAs}$ for decay and regulates cell motility in mouse myoblasts. PLoS Genet. 2012;8(8):e1002901.

18. Devany E, Zhang X, Park JY, Tian B, Kleiman FE. Positive and negative feedback loops in the p53 and mRNA 3' processing pathways. Proc Natl Acad Sci U S A. 2013;110(9):3351-3356.

19. Ceccaldi R, et al. Bone marrow failure in Fanconi anemia is triggered by an exacerbated p53/p21 DNA damage response that impairs hematopoietic stem and progenitor cells. Cell Stem Cell. 2012;11(1):36-49.

20. Dumitriu B, Young NS. Damage control and its costs: BM failure in Fanconi anemia stems from overactive p53/p21. Cell Stem Cell. 2012;11(1):7-8.

21. Boultwood J, Pellagatti A, Wainscoat JS. Haploinsufficiency of ribosomal proteins and p53 activation in anemia: Diamond-Blackfan anemia and the 5q- syndrome. Adv Biol Regul. 2012;52(1):196-203.

22. Ogden GR, Lane DP, Chisholm DM. p53 expression in dyskeratosis congenita: a marker for oral premalignancy? J Clin Pathol. 1993;46(2):169-170.

23. Westin ER, Aykin-Burns N, Buckingham EM, Spitz DR, Goldman FD, Klingelhutz AJ. The p53/p21(WAF/CIP) pathway mediates oxidative stress and senescence in dyskeratosis congenita cells with telomerase insufficiency. Antioxid Redox Signal. 2011;14(6):985-997.

24. Simeonova I, et al. Mutant mice lacking the p53 C-terminal domain model telomere syndromes. Cell Rep. 2013;3(6):2046-2058. 